\title{
The crystal structure of four solvates of a bisphenol derivative
}

\author{
Fujino Mizuki, ${ }^{1}$ Saori Gontani, ${ }^{1}$ Takeshi Nakamura, ${ }^{2}$ Shinya Matsumoto ${ }^{1}$ \\ ${ }^{I}$ Graduate School of Environment and Information Sciences, Yokohama National University \\ ${ }^{2}$ Mitsubishi Chemical Corporation Osaka $R \& D$ Center \\ matsumoto-shinya-py@ynu.ac.jp
}

The physicochemical properties of solvated crystals are greatly influenced by the interactions between the solvent and solute. Therefore, various properties of solvated crystals, such as stability, spectral properties, and solubility, are variable depending on the type of solvent molecules included in the lattice. The design of solvated crystallization has attracted great attention as a means of modifying the properties of organic solids, especially in the fields of pharmaceuticals and organic optoelectronic materials. [1][2] Solvates formation of organic compounds is also important in the separation process in chemical industries. [3]

Bisphenol A derivatives are applied as a raw material of polymers for various applications. Solvates formation is applied in the process of industrial production of several bisphenol A derivatives. The bisphenol A derivative 1 shown in Fig. 1 (a) was found to form solvates with branched alcohols, but it was difficult to form solvates with linear alcohols. To clarify the observed difference in its solvation behavior, X-ray structure analysis was performed on its solvates with isopropanol (IPA), 2-butanol (2-BuOH), iso-butanol $(i-\mathrm{BuOH})$, and water.

The result showed that the four solvated crystals involve $\mathbf{1}$ and a solvent molecule in a 1:1 ratio. The four solvated crystals were also found to be isomorphous. One solvent molecule in the crystals is linked to two neighboring molecules of $\mathbf{1}$ by intermolecular hydrogen bonds along the $a$-axis and included in the void surrounded by the bisphenol moieties. There found no significant structural difference in the arrangement of $\mathbf{1}$ itself in the four solvates, whereas the space around the solvent molecules was dependent on the size of a solvent molecule. To compare the differences between the space for solvent molecules, the three interatomic distances $X, Y$, and $Z$ shown in Fig. 1 (b) were examined. The structure of the solvated crystals was found to be expanded along the $Y$ direction due to the solvent molecules, whereas there was no remarkable difference in the other two directions as listed in Table 1. This structural feature could be correlated with the crystallization behavior of these solvates and their stability.

(a)

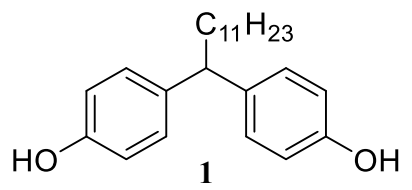

(b)

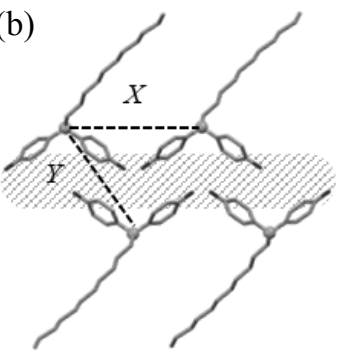

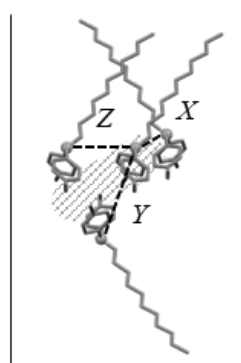

Figure 1. (a) Molecular structure of 1, and (b) three geometrical parameters $X, Y$, and $Z$.

Table 1. Three distances in the four solvates.

\begin{tabular}{clcl}
\hline Solvate & \multicolumn{3}{c}{ Distance / $\AA$} \\
& $X$ & $Y$ & $Z$ \\
\hline $\mathrm{H}_{2} \mathrm{O}$ & $11.110(2)$ & $10.350(2)$ & $5.886(2)$ \\
$\mathrm{PA}$ & $11.369(2)$ & $13.013(2)$ & $5.913(2)$ \\
$2-\mathrm{BuOH}$ & $11.333(2)$ & $13.249(2)$ & $6.045(3)$ \\
$i-\mathrm{BuOH}$ & $11.171(3)$ & $14.025(3)$ & $5.984(3)$ \\
\hline
\end{tabular}

[1] Steed, J. W., Trends in Pharmacological Sciences (2013), 34, 185.

[2] Dongpeng Y., Amit D. et al., Angew Chem. (2011), 50, 12483.

[3] Mitsubishi Chemical Corp., Hayashi K., Harada T. \& Nakamoto M., Production of Bisphenol A, JP2000327614, (2000).

\section{Keywords: Bisphenol A, Functional molecule, X-ray structure analysis, Solvates}

